\section{Abortion Safety and Use with Normally Prescribed Mifepristone in Canada}

\author{
Laura Schummers, Sc.D., Elizabeth K. Darling, Ph.D., Sheila Dunn, M.D., \\ Kimberlyn McGrail, Ph.D., Anastasia Gayowsky, M.Sc., Michael R. Law, Ph.D., \\ Tracey-Lea Laba, Ph.D., Janusz Kaczorowski, Ph.D., \\ and Wendy V. Norman, M.D., M.H.Sc.
}

\section{A BSTRACT}

\section{BACKGROUND}

In the United States, mifepristone is available for medical abortion (for use with misoprostol) only with Risk Evaluation and Mitigation Strategy (REMS) restrictions, despite an absence of evidence to support such restrictions. Mifepristone has been available in Canada with a normal prescription since November 2017.

\section{METHODS}

Using population-based administrative data from Ontario, Canada, we examined abortion use, safety, and effectiveness using an interrupted time-series analysis comparing trends in incidence before mifepristone was available (January 2012 through December 2016) with trends after its availability without restrictions (November 7, 2017, through March 15, 2020).

\section{RESULTS}

A total of 195,183 abortions were performed before mifepristone was available and 84,032 after its availability without restrictions. After the availability of mifepristone with a normal prescription, the abortion rate continued to decline, although more slowly than was expected on the basis of trends before mifepristone had been available (adjusted risk difference in time-series analysis, 1.2 per 1000 female residents between 15 and 49 years of age; 95\% confidence interval [CI], 1.1 to 1.4), whereas the percentage of abortions provided as medical procedures increased from $2.2 \%$ to $31.4 \%$ (adjusted risk difference, 28.8 percentage points; 95\% CI, 28.0 to 29.7). There were no material changes between the period before mifepristone was available and the nonrestricted period in the incidence of severe adverse events ( $0.03 \%$ vs. $0.04 \%$; adjusted risk difference, 0.01 percentage points; $95 \% \mathrm{CI},-0.06$ to 0.03$)$, complications $(0.74 \%$ vs. $0.69 \%$; adjusted risk difference, 0.06 percentage points; $95 \% \mathrm{CI},-0.07$ to 0.18$)$, or ectopic pregnancy detected after abortion $(0.15 \%$ vs. $0.22 \%$; adjusted risk difference, -0.03 percentage points; $95 \% \mathrm{CI},-0.19$ to 0.09). There was a small increase in ongoing intrauterine pregnancy continuing to delivery (adjusted risk difference, $0.08 \%$; $95 \% \mathrm{CI}, 0.04$ to 0.10 ).

\section{CONCLUSIONS}

After mifepristone became available as a normal prescription, the abortion rate remained relatively stable, the proportion of abortions provided by medication increased rapidly, and adverse events and complications remained stable, as compared with the period when mifepristone was unavailable. (Funded by the Canadian Institutes of Health Research and the Women's Health Research Institute.)
From the Department of Family Practice (L.S., W.V.N.) and the Centre for Health Services and Policy Research, School of Population and Public Health (K.M., M.R.L.), University of British Columbia, Vancouver, ICES (L.S., E.K.D., A.G.) and the Department of Obstetrics and Gynecology (E.K.D.), McMaster University, Hamilton, ON, the Department of Family and Community Medicine, University of Toronto, and the Women's College Research Institute, Women's College Hospital, Toronto (S.D.), and the Department of Family and Emergency Medicine, University of Montreal, Montreal (J.K.) - all in Canada; the Centre for Health Economics Research and Evaluation, University of Technology, Sydney (T.-L.L.); and the Department of Public Health, Environments, and Society, Faculty of Public Health and Policy, London School of Hygiene and Tropical Medicine, London (W.V.N.). Dr. Norman can be contacted at wendy.norman@ubc.ca or at the Department of Family Practice, University of British Columbia, 5950 University Blvd., Vancouver, BC, V6T 1Z3, Canada.

This article was published on December 8 , 2021, at NEJM.org.

DOI: 10.1056/NEJMsa2109779 Copyright $\odot 2021$ Massachusetts Medical Society. 
CCESS TO SAFE ABORTION IS A HUMAN right and a key component of reproductive health, yet inadequate access remains a global concern. ${ }^{1}$ A medical abortion regimen of mifepristone and misoprostol has been shown to be safe..$^{2-4}$ Mifepristone is approved for use in the United States with Risk Evaluation and Mitigation Strategy (REMS) restrictions ${ }^{5}$ (including mandatory prescriber certification, observed dosing, dispensing by the prescriber or medical facility with the exclusion of pharmacies, and submission of a prespecified patient consent form) and elsewhere with similar restricted approvals. ${ }^{6,7}$ Professional organizations have called for the removal of REMS restrictions because they impede access to abortion services without improving safety. ${ }^{8}$ However, high-quality data with respect to abortion safety and effectiveness when mifepristone is available without REMS-like restrictions are lacking.'

Mifepristone was first marketed in Canada in January 2017 as a 200-mg tablet combined with $800 \mu \mathrm{g}$ of misoprostol. ${ }^{10}$ Approval came more than 15 years after approval in the United States and more than 25 years after similar rulings in France, Sweden, and the United Kingdom. ${ }^{11}$ Initially, regulatory restrictions in Canada were similar to REMS restrictions. ${ }^{12}$ By November 7, 2017, Canadian regulators had removed these restrictions so that mifepristone could be prescribed and dispensed as a normal prescription medication and had expanded approved use from 49 to 63 days after the patient's last menstrual period. ${ }^{13}$ This action resulted in a globally unprecedented practice of permitting any physician or nurse practitioner to prescribe, any pharmacist to dispense, and patients to independently administer mifepristone when, where, and if they chose. ${ }^{14}$ Before 2017, medically induced abortions made up only $4 \%$ of all abortions in Canada and used off-label regimens of misoprostol with or without methotrexate. These regimens have reduced effectiveness (84 to $97 \%$ ) and a high risk of teratogenicity if the abortion fails. ${ }^{4,15}$

We compared abortion use, safety, and effectiveness during the period after mifepristone had become available without REMS-like restrictions with the period before mifepristone had been available in Ontario, Canada (representing nearly $40 \%$ of the Canadian population).
METHODS

\section{DATA SET}

In Canada, universal single-payer health care including coverage for abortion services and management of its complications - is provided by each province or territory. We used linked administrative health data ${ }^{16}$ to create a populationbased cohort of all female Ontario residents between the ages of 12 and 49 years who had received abortion services from January 1, 2012, to March 15, 2020. We linked records from practitioner visits, all hospital visits, and outpatient prescriptions using a secure data platform at ICES (formerly known as the Institute for Clinical Evaluative Sciences) at McMaster University. ${ }^{16,17}$ We excluded events that had occurred within 6 weeks before or after a missed abortion (pregnancy loss without expulsion) or spontaneous abortion (pregnancy loss with expulsion) and those occurring within 6 weeks after delivery at 25 weeks or more of gestation to avoid including procedures that could have been misclassified as abortions. Details regarding the data set are provided in Figure S1 and Table S1 in the Supplementary Appendix, available with the full text of this article at NEJM.org. Ethics approval for the study was granted by the University of British Columbia.

\section{EXPOSURE AND OUTCOMES}

The exposure we examined was the regulatory change that made mifepristone available as a normal prescription. Outcomes included measures of abortion use, safety, and effectiveness.

We evaluated outcomes regarding abortion use that included the abortion rate, which was calculated according to the international standard as the annual number of abortions among female residents between 15 and 49 years of age per 1000 female residents in that age group ${ }^{18}$ the percentage of all abortions that were medically induced, and the percentage of all abortions that were provided at 14 weeks or more of gestation (second-trimester abortion). (In the calculation of the abortion rate, the lower age for female residents was 15 years, as compared with a lower age of 12 years that was used for all other calculations in our study cohort.) Abortion safety outcomes within 6 weeks after abortion were severe adverse 
events, including any blood transfusion, abdominal surgery (laparotomy, laparoscopy, or hysterectomy), admission to an intensive care unit, or sepsis that occurred during a hospitalization associated with an abortion-complication code. Complications of abortion included genital tract or pelvic infection, hemorrhage (delayed or excessive bleeding that complicated complete or incomplete abortion), embolism, shock, renal failure, damage to pelvic organs or tissues (including uterine perforation), venous complications, and other or unspecified complications. Outcomes regarding abortion effectiveness were the incidence of subsequent uterine evacuation (aspiration after medical abortion, reaspiration after surgical abortion, or subsequent abortion procedure), ongoing intrauterine pregnancy continuing until delivery, and ectopic pregnancy diagnosed within 6 weeks after the abortion date. Detailed outcome definitions are provided in Table S1.

\section{STATISTICAL ANALYSIS}

We tabulated the incidence of each outcome according to the mifepristone regulatory period. We then conducted interrupted time-series analysis using segmented generalized mixed-effects regression to compare the expected incidence and trend for each outcome based on the period before mifepristone had become available with the observed level and trend after the availability of mifepristone with a normal prescription. We used log binomial regression to model incidence outcomes and Poisson regression with population offset to calculate the abortion rate; models were adjusted for outcome trends before the approval of mifepristone and accounted for autocorrelation and correlated residuals (File 1 in the Supplementary Appendix). ${ }^{19,20}$ We used 6-month moving averages to smooth the resulting estimates. We examined outcomes from January 1, 2017, through November 6, 2017, descriptively but excluded this period from our models because it included rapid, incremental regulatory changes. ${ }^{13}$

We graphed the observed and expected monthly outcome incidence (quarterly for outcomes with $<6$ events in any month) following best practices. ${ }^{21}$ We estimated risk differences and risk ratios for each outcome by comparing the observed with expected values for September 2019, a time point selected a priori to balance model stability (greatest in the middle of the study period) and integration of mifepristone into practice (greatest at the end of the study period). We used bootstrapping with 200 samples drawn with replacement to estimate $95 \%$ confidence intervals ${ }^{22}$ without adjustment for multiple comparisons. All analyses were conducted with the use of SAS software, version 7.51, and R software (code in File 2 in the Supplementary Appendix).

To examine the robustness of our findings to modeling specification, we conducted sensitivity analyses using segmented generalized leastsquares regression, with autocorrelation terms selected on the basis of the Durbin-Watson test $\mathrm{t}^{23-25}$ and visual examination of autocorrelation function and partial autocorrelation function residuals. ${ }^{25}$ We conducted subgroup analyses that were restricted to first-trimester abortions and then further restricted to first-trimester medical abortions.

RESULTS

CHARACTERISTICS OF THE STUDY POPULATION

Of the 314,859 induced abortions in Ontario, Canada, from January 1, 2012, through March 15, 2020, the majority (89.3\%) were surgical (with $94.6 \%$ performed by means of suction aspiration), approximately $10 \%$ were medical abortions, and less than $0.1 \%$ were unclassified. Table 1 shows cohort characteristics according to the regulatory period for mifepristone.

\section{DESCRIPTIVE ANALYSES OF ABORTION OUTCOMES}

The abortion rate per 1000 female residents of reproductive age and the incidence of all other outcomes are presented descriptively according to the regulatory period in Table 2. (Components of the composite outcomes are shown in Table S2.) The abortion rate decreased from 11.9 abortions per 1000 female residents between the ages of 15 and 49 years of age before mifepristone had become available to 11.3 per 1000 female residents after mifepristone had become available with a normal prescription. The percentage of all abortions that were provided medically increased from $2.2 \%$ before mifepristone had become available to $8.3 \%$ while mifepristone was restricted and then to $31.4 \%$ after mifepristone had become available with a normal prescription. The rate of second-trimester abortions declined from 5.5\% of all abortions to $5.1 \%$ after the availability of mifepristone with a normal prescription. 


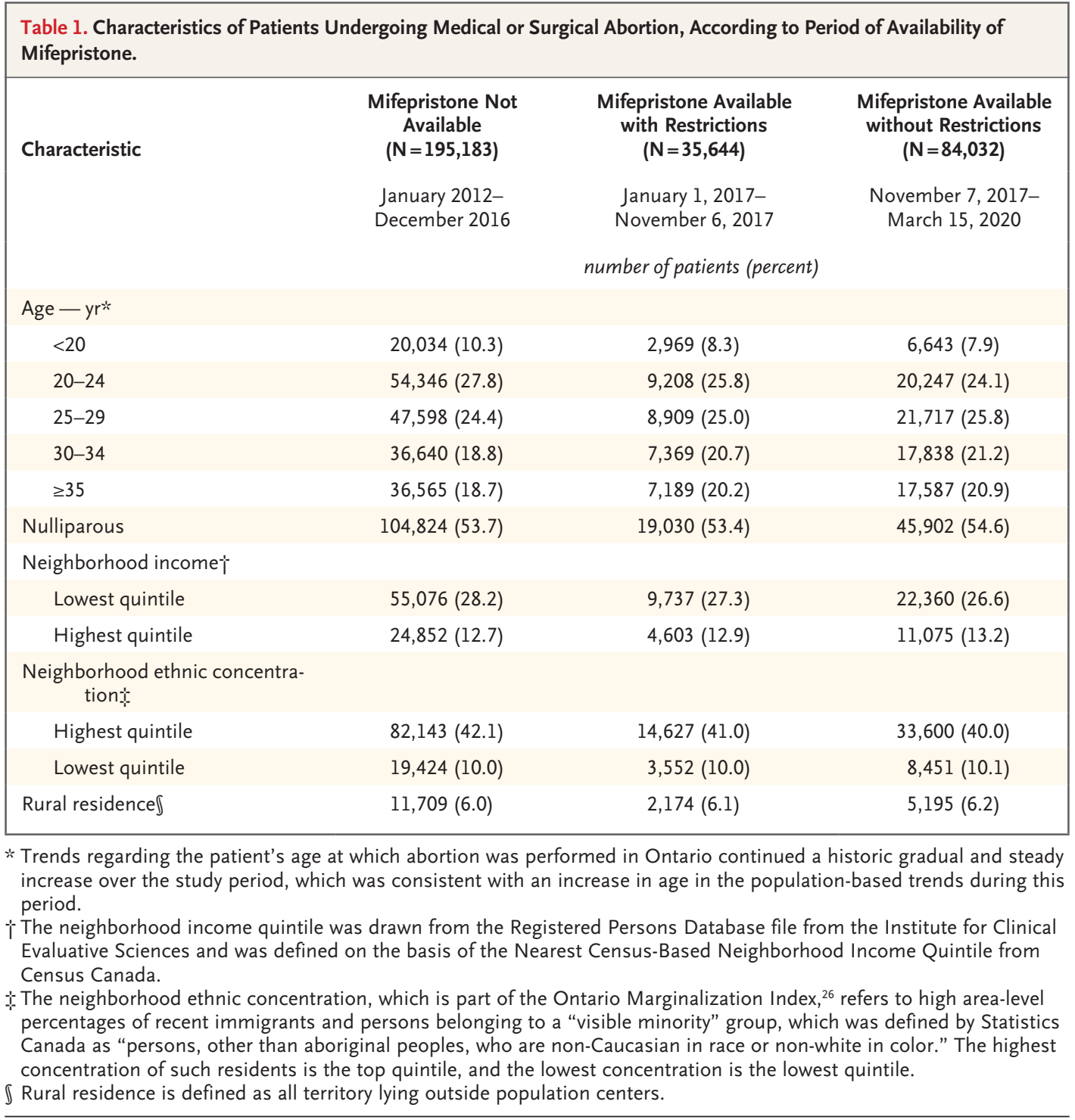

Abortion safety outcomes remained stable during the period before mifepristone had become available and during the period after its availability with a normal prescription (severe adverse events, $0.03 \%$ and $0.04 \%$, respectively; and abortion complications, $0.67 \%$ and $0.74 \%$, respectively). Subsequent uterine evacuation increased from $1.0 \%$ to $2.2 \%$, and ongoing intrauterine pregnancy continuing until delivery increased from $0.03 \%$ to $0.08 \%$. Ectopic pregnancy that was detected after abortion increased from $0.15 \%$ to $0.22 \%$.

TIME-SERIES ANALYSES OF ABORTION OUTCOMES

Interrupted time-series graphs of abortion-use outcomes are presented in Figure 1, abortion safety outcomes in Figure 2, and abortion-effectiveness outcomes in Figure 3. Adjusted risk differences and risk ratios from these models comparing the period before mifepristone had become available with the nonrestricted period are presented in Table 2 .

During the study period, the abortion rate continued an absolute decline, although as compared with the trend before the approval of mifepristone, we noted an increase of 1.2 abortions per 1000 female residents $(95 \%$ confidence interval [CI], 1.1 to 1.4 ) over the predicted rate. The proportion of all abortions that were medical increased by an adjusted risk difference of 28.8 percentage points (95\% CI, 28.0 to 29.7). 


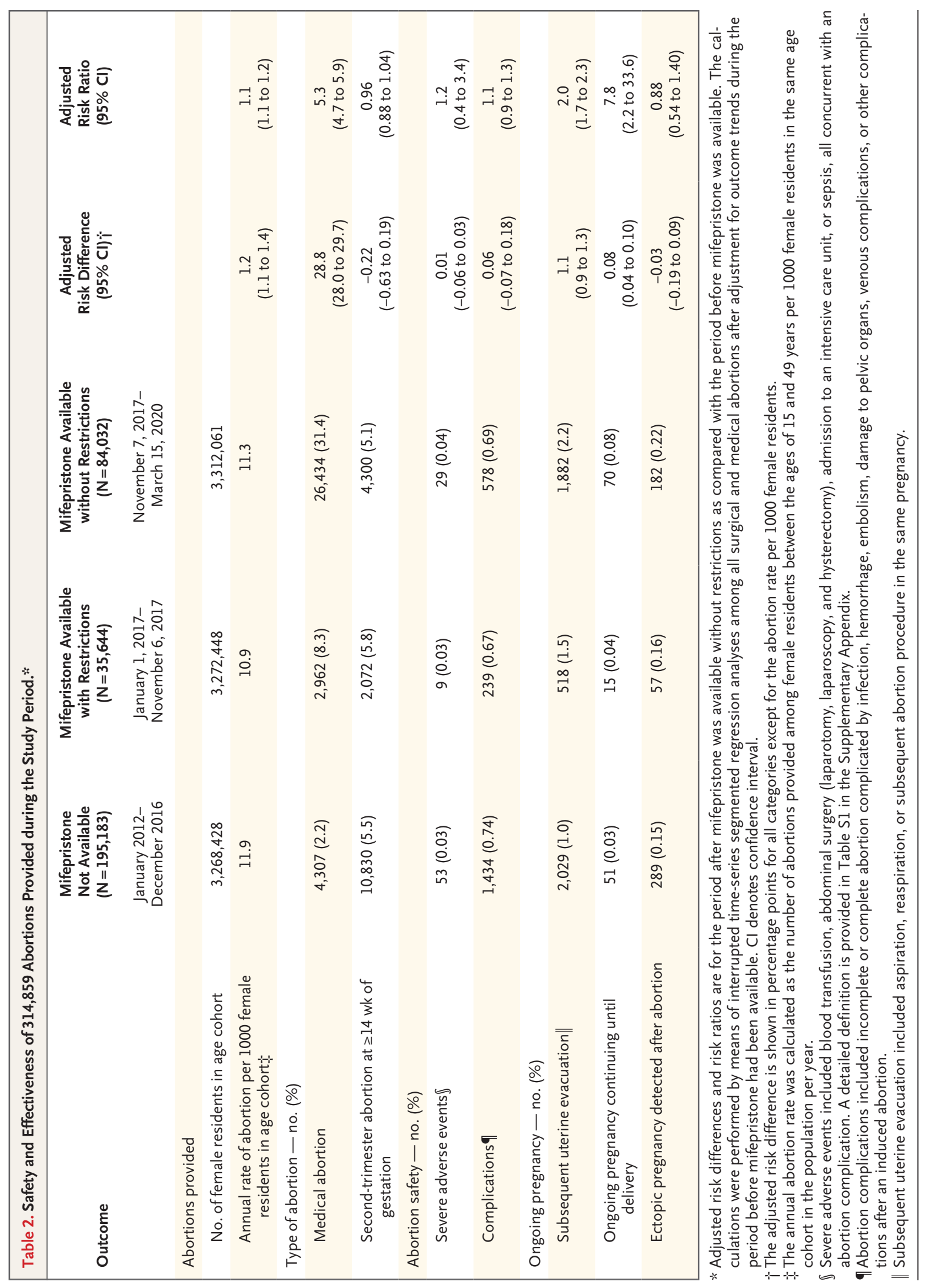


_ Observed model - - - - Expected trend before mifepristone approval

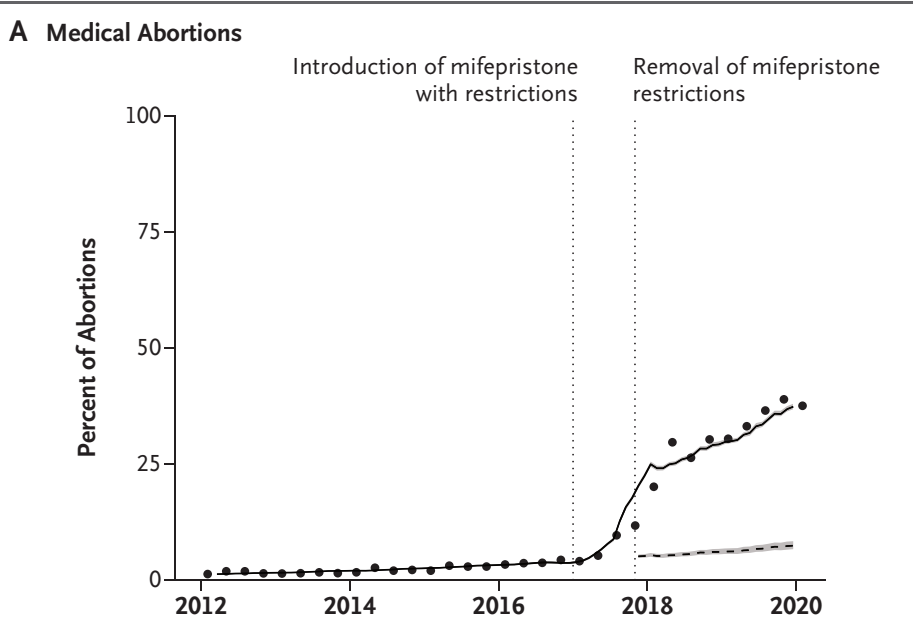

B Abortion Rate among Female Residents 15-49 Yr of Age

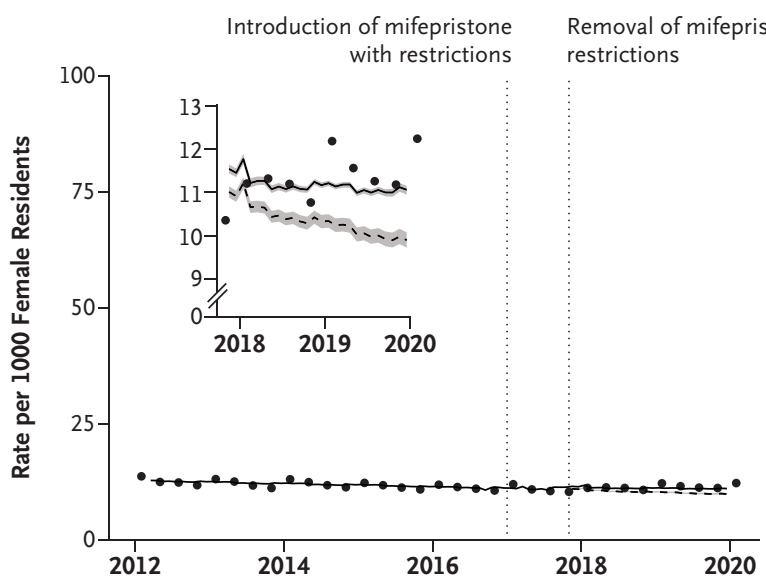

C Second-Trimester Abortions

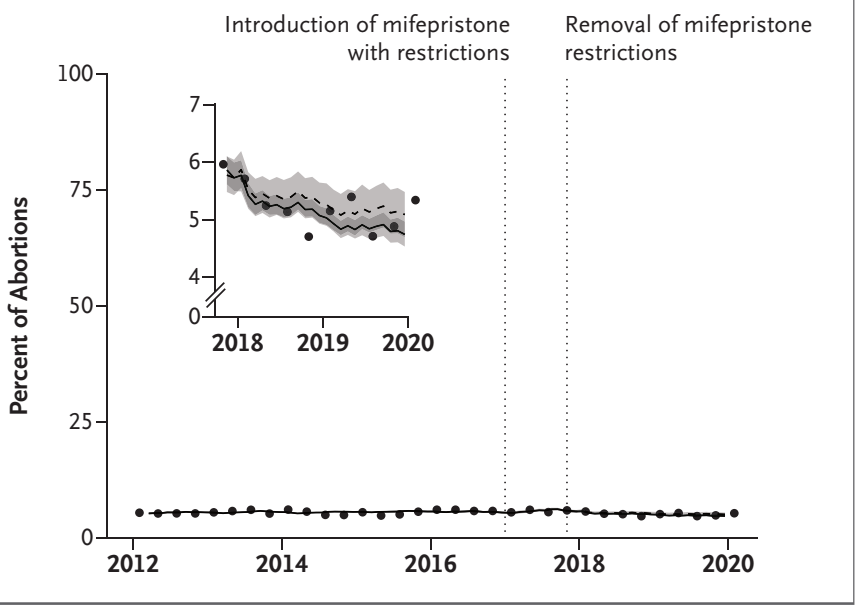

Figure 1. Changes in the Percentages of Medical and Second-Trimester Abortions among All Abortions and in Abortion Rates.

Shown are the results of interrupted time-series analyses of the level and trend of abortion outcomes in Ontario, Canada, among all surgical and medical abortions that were provided before the introduction of mifepristone in the province (2012 through 2016), after the introduction but with Risk Evaluation and Mitigation Strategy (REMS)-like restrictions (January 1, 2017, through November 6, 2017), and after a regulatory change to remove restrictions, which made mifepristone available by normal prescription (November 7, 2017, through March 15, 2020). Panel A shows the percentage of all abortions that were performed medically at any gestational age. Panel B shows the annual abortion rate among female residents between the ages of 15 and 49 years per 1000 female residents in the same age group in the population. Panel $\mathrm{C}$ shows the percentage of second-trimester abortions ( $\geq 14$ weeks of gestation) among all abortions. In Panels B and C, the insets show the same data on an expanded $y$ axis; shading indicates $95 \%$ confidence intervals. The expected outcomes if mifepristone had not been available were estimated from segmented mixed-effects models (log binomial regression in Panels $A$ and $C$ and Poisson regression with population offset in Panel B) and smoothed with the use of a 6-month moving-average function.

The rate of second-trimester abortions showed a stable, continuous decline (adjusted risk difference, -0.22 percentage points; $95 \% \mathrm{CI},-0.63$ to 0.19). Abortion safety outcomes were materially stable, with an adjusted risk difference of 0.01 percentage points ( $95 \% \mathrm{CI},-0.06$ to 0.03 ) for severe adverse events and 0.06 percentage points ( $95 \%$ CI, -0.07 to 0.18 ) for complications. The rate of subsequent uterine evacuation increased modestly, with an adjusted risk difference of 1.1 percentage points ( $95 \% \mathrm{CI}, 0.91$ to 1.3), and the rate of ongoing intrauterine pregnancy that continued until delivery increased by 0.08 percentage points ( $95 \% \mathrm{CI}, 0.04$ to 0.10 ). The rate of ectopic pregnancy that was detected after abortion was materially stable, with an adjusted risk difference of -0.03 percentage points $(95 \% \mathrm{CI}$, -0.19 to 0.09 ).

Interrupted time-series graphs from generalized least-squares regression with the use of aggregated monthly data showed the robustness of the findings to modeling specification (Figs. 
S2, S3, and S4). Changes in outcome incidences and trends after mifepristone availability with a normal prescription were consistent for all outcomes except for the percentage of second-trimester abortions, for which aggregated models indicated a slight reduction $(-0.92$ percentage points; $95 \% \mathrm{CI},-1.40$ to -0.48$)$.

\section{OUTCOMES AFTER FIRST-TRIMESTER ABORTION}

Outcome incidences among all first-trimester abortions are presented in Tables $\mathrm{S} 3$ and $\mathrm{S} 4$ and Figures S5, S6, and S7; outcomes among firsttrimester medical abortions are provided in Tables S5 and S6. The percentage of first-trimester abortions that were performed medically increased from $1.6 \%$ before mifepristone was available to $32.4 \%$ after mifepristone was available without restrictions. Severe adverse events were rare among first-trimester medical abortions $(<6$ events per 25,744 abortions [too infrequent to report exact incidence]), the incidence of abortion complications was $0.76 \%$, and the incidence of subsequent uterine evacuation was $4.5 \%$. Similarly, ongoing intrauterine pregnancy was uncommon, with $0.13 \%$ continuing to delivery. Ectopic pregnancy detected after abortion that occurred with any severe adverse event was also rare $(<6$ per 314,859 abortions).

\section{DISCUSSION}

We comprehensively examined changes in abortion use, safety, and effectiveness during the period when mifepristone had become available without REMS-like restrictions in a populationbased cohort of abortion service users in Ontario, Canada. We found that after mifepristone had become available with a normal prescription dispensed by pharmacists and taken at user discretion, abortion rates were materially stable, medical abortion uptake was rapid, and abortion-related adverse events and ectopic pregnancy remained rare, as compared with before mifepristone had been available.

The modestly slower decline in the abortion rate, relative to the expected decline based on the trend before mifepristone had become available, may be due in part to the provision of abortion earlier in pregnancy. Since 4 to $7 \%$ of pregnancies per week in the first trimester ${ }^{27}$ end in spontaneous abortion, the availability of abortion at earlier gestational ages would increase the abor-

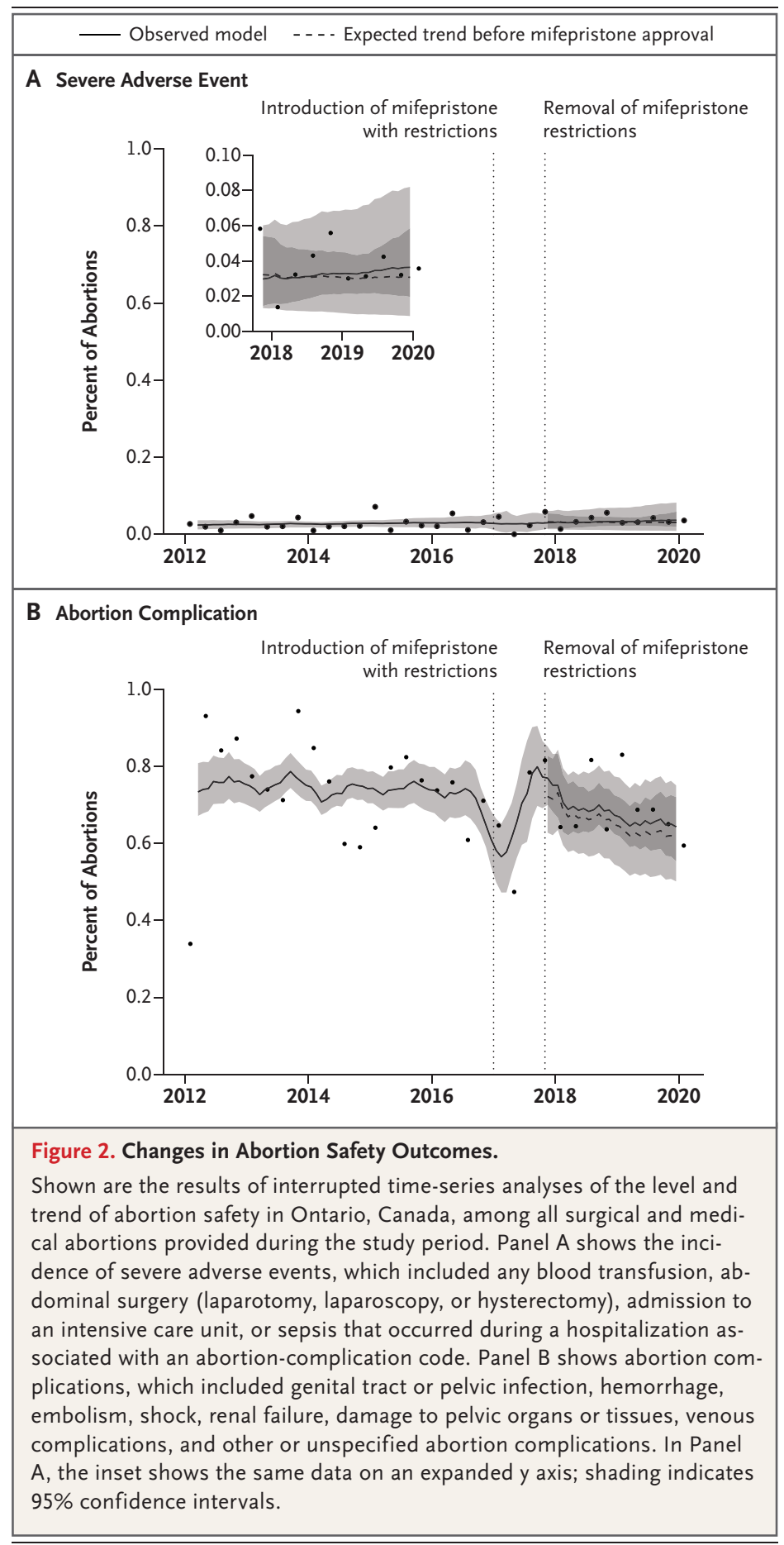

tion rate by enabling termination of pregnancy before the occurrence of miscarriage, even in the absence of a true increase in demand for abortion. The availability of mifepristone without restrictions may have slowed the decline in the abortion rate through improved abortion access, a 


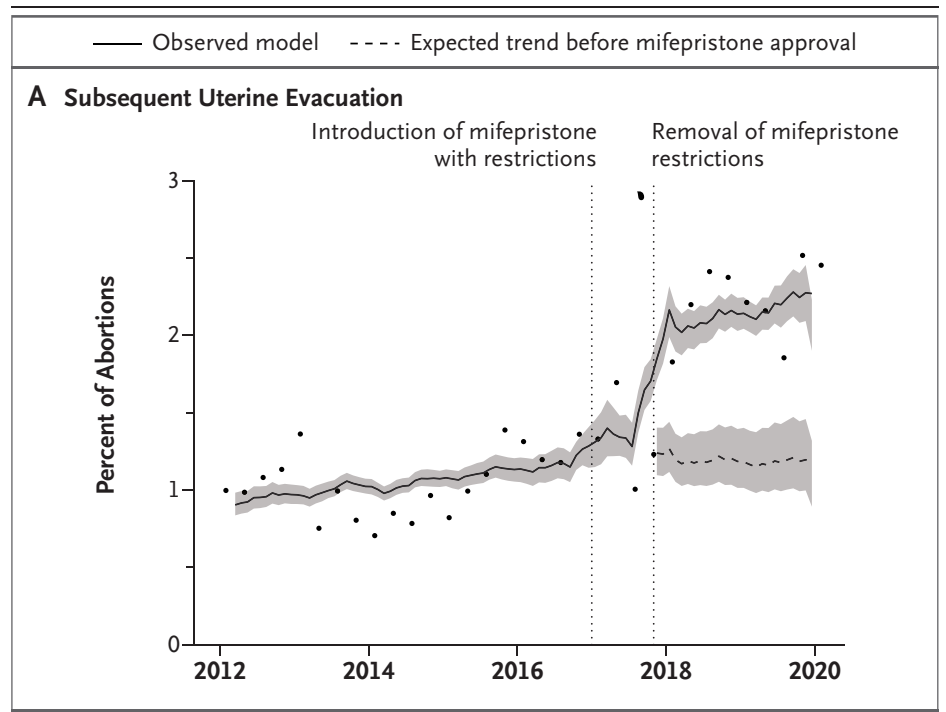

B Subsequent Delivery

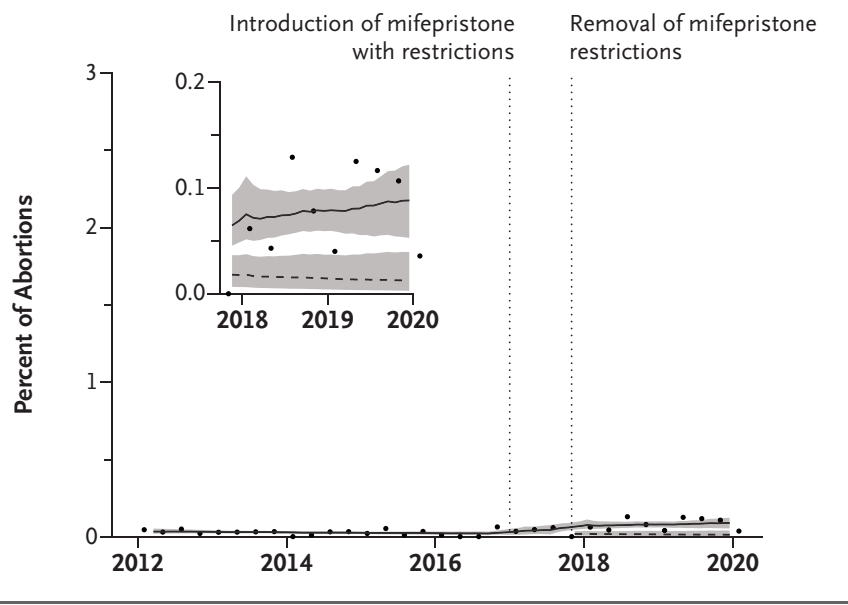

C Ectopic Pregnancy Detected after Abortion

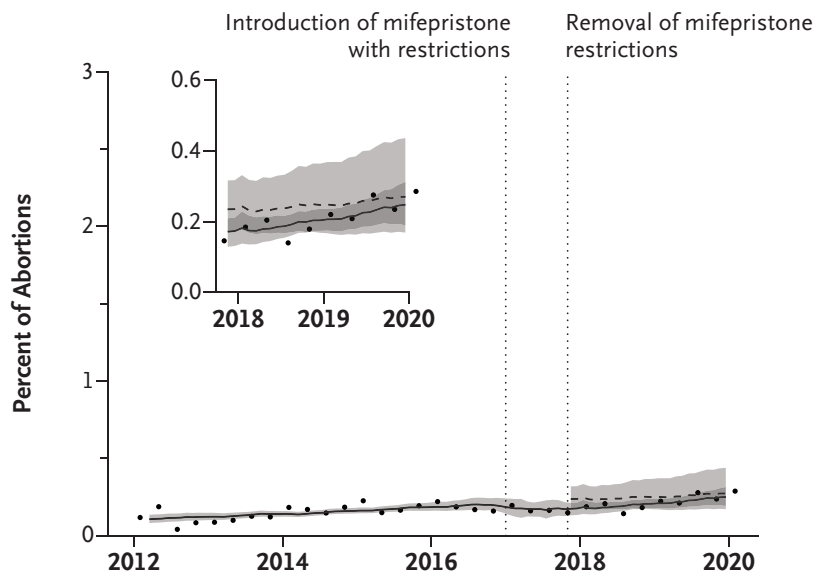

Figure 3. Abortion Effectiveness and Ongoing Pregnancy Outcomes.

Shown are the results of interrupted time-series analyses of the level and trend of ongoing pregnancy outcomes among all surgical and medical abortions provided during the study period. These outcomes include the incidences of uterine evacuation after abortion (Panel $A$ ), ongoing intrauterine pregnancy continuing until delivery (Panel B), and ectopic pregnancy detected after abortion (Panel C). Shading indicates $95 \%$ confidence intervals.

hypothesis that is consistent with findings that restrictive policies regarding the prescription of mifepristone worsen access to abortion ${ }^{28}$ and that abortion rates increase when access improves. ${ }^{29}$ Because we did not measure pregnancy intention in our study, we cannot differentiate trend changes in unintended pregnancy from changes in the fraction of pregnancies that were terminated. Our findings indicate that improved abortion access was not associated with a material increase in the abortion rate.

The uptake of mifepristone for medical abortion under Canada's unrestricted regulations was faster than reported in settings with restrictive regulations. Although more than one third of abortions in Ontario were medically induced 2 years after mifepristone had been available as a normal prescription, $5.2 \%$ of abortions in the United States were medically induced 2 years after mifepristone availability, with the percentage slowly increasing to $39.0 \% 17$ years after availability. ${ }^{2}$ Similarly slower uptake has been reported in European settings that have mifepristone restrictions, even among those where mifepristone had been introduced long after best practice guidelines had been established. ${ }^{30}$

Our findings indicate that abortion remained safe and ongoing pregnancy remained infrequent after unrestricted access to mifepristone. Without observed administration, some patients with a prescription for mifepristone may have never used it. ${ }^{9}$ However, the infrequent occurrence of ongoing intrauterine pregnancy indicates that patients who received mifepristone most often correctly used the medication without supervision..$^{31}$ Our abortion safety and effectiveness findings are consistent with the results 
of recent studies examining patient-reported outcomes during the coronavirus disease 2019 pandemic, when REMS-like restrictions were temporarily removed in some settings. ${ }^{9,32,33}$ A study involving 52,142 patients in the United Kingdom showed no material differences in success rates or serious adverse events between abortions provided under REMS-like restrictions and a telemedicine-hybrid model with investigations such as ultrasonography performed only when indicated. ${ }^{33}$

The small increase in the incidence of ectopic pregnancy that was detected after unrestricted access to mifepristone was consistent with the increasing trend before the availability of mifepristone, which indicated no increase over the expected incidence. A 2012 cross-sectional survey of abortion providers in the United States and Canada showed that more than $90 \%$ of providers routinely performed ultrasonography before abortion, ${ }^{34}$ even though the value of such imaging in the absence of known ectopic risks or symptoms had not been shown. ${ }^{2,35}$ Ectopic pregnancy is more likely to be detected after abortion that is provided at earlier stages of gestation before a clinical or ultrasonographic diagnosis. Because undiagnosed ectopic pregnancy can lead to tubal rupture and death, ${ }^{36}$ identifying ectopic pregnancy before the onset of complications with the use of clear clinical protocols ${ }^{2,4}$ is essential, although such procedures do not need to be performed before the initiation of medical abortion. ${ }^{33}$

Our safety and ongoing pregnancy findings among first-trimester medical abortions during the period after unrestricted access to mifepristone were consistent with reports from other settings with restricted access. ${ }^{2,4}$ In settings with REMS-like restrictions, first-trimester medical abortions resulted in major adverse events in 0.3 to $0.5 \%$ of women ${ }^{2,431}$ and blood transfusion in 0.04 to $0.10 \%{ }^{4,31}$ Among medical abortions performed up to 63 days after the last menstrual period, subsequent uterine evacuation occurred in 2.0 to $4.8 \%$ of patients and ongoing intrauterine pregnancy in 0.5 to $2.0 \% .^{2,4}$ In our study among first-trimester abortions, severe adverse events were too infrequent to report an incidence value, $0.04 \%$ of the patients underwent blood transfusion, $4.5 \%$ underwent uterine evacuation, and $0.13 \%$ had an ongoing pregnancy continuing to delivery. Although the incidence of uterine evacuation was increasing before mifepristone had become available, the expected incidence trend after the availability of mifepristone leveled off because of the more rapid increase in the number of abortions (the denominator). Because subsequent uterine evacuation is substantially more frequent after medical abortion than after surgical procedures $(<3.0 \%),{ }^{4,37}$ a practice shift to more medical abortions is expected to increase the incidence of this outcome.

Our study has several potential limitations. The fundamental assumption underlying the validity of interrupted time-series analysis is that outcome trends before the exposure of interest would have continued if the exposure had not occurred. This assumption does not hold if other policy, practice, or contextual changes that may have an effect on outcome incidence occur concurrent to the exposure of interest. ${ }^{20}$ However, this analytic approach is robust with respect to changes in the individual-level characteristics of patients or provider practices that accrue gradually over the study period, since such changes are accounted for in trend regression terms during the period before mifepristone had become available. Careful review of policies, academic literature, practice guidelines, and media output that are related to abortion during the study period identified no concurrent changes that would have invalidated our analytic approach. Practitioner fees, training programs, administrative data codes, and cost coverage for the drug were stable during the study period. Surveys and interviews among practitioners indicate that initial mifepristone restrictions were barriers to broad adoption of this practice. ${ }^{13,38}$ The short period during which mifepristone was available in Canada with REMS-like restrictions (January 1 to November 6, 2017) precludes a formal analysis of mifepristone availability with restrictions as compared with such availability without restrictions. The unrestricted availability of mifepristone appears to be the fundamental factor associated with changes in our study outcomes.

Our prescription database universally captured mifepristone prescriptions that were dispensed after August 10, 2017 (when a universal no-cost subsidy was introduced) but only captured mifepristone prescriptions from January to August 9, 2017, among patients with incomebased prescription subsidies and those under 25 years of age. These factors may have contributed to an underestimation of early mifepristone up- 
take. However, this limitation was mitigated by our identification of medical abortions using data regarding practitioner payments, procedures, and prescriptions, along with our exclusion of these months from our time-series analysis. Our population-based data comprehensively captured all abortions among Ontario residents, as well as all subsequent hospital or health service events, even if such services were not provided by the same provider or facility that provided the initial care. Therefore, loss to follow-up was minimal since it involved only patients who had moved out of the province within 6 weeks after the abortion or during the current pregnancy. However, since linkages across databases are possible only for residents who are eligible for provincial health insurance, we excluded the 397 abortions (0.1\%) that were provided to nonresidents. Because of lags in availability of cause-of-death data, we could not report the incidence of abortion-related deaths. However, surveillance by the U.S. Centers for Disease Control and Prevention indicates that death is a very rare outcome (2 deaths among 609,095 abortions in 2018)..$^{39}$ Although minimal data were missing for gestational trimester, we did not have data regarding specific gestational ages in weeks, which prevented an evaluation of changes to abortion timing within trimesters.

When mifepristone became available as a normally prescribed medication in Canada, the frequency of medical abortion rose substantially as compared with the frequency during the period before mifepristone became available, even though the rate of abortion remained materially stable. The incidences of serious adverse events and complications remained materially unchanged, and uterine evacuation and ongoing pregnancy remained infrequent.

Parts of this material are based on data and information compiled and provided by the Ontario Ministry of Health and the Canadian Institute for Health Information. The analyses, conclusions, opinions, and statements expressed in this article are solely those of the authors and do not reflect those of the funding or data sources.

Supported by a grant (PJT-168964) from the Canadian Institutes of Health Research and by a grant from the Women's Health Research Institute of the Provincial Health Services Authority of British Columbia.

Disclosure forms provided by the authors are available with the full text of this article at NEJM.org.

We thank staff members at ICES, which is supported by the Government of Ontario Ministry of Health and the Ministry of Long-Term Care, for their assistance with data management; staff members at IQVIA Solutions Canada for use of their Drug Information File; and our fellow members of the Canadian Contraception and Abortion Research Team for their contributions to the study.

\section{REFERENCES}

1. United Nations High Commissioner for Human Rights. Information series on sexual and reproductive health and rights: abortion. 2020 (https://www.ohchr.org/ Documents/Issues/Women/WRGS/ SexualHealth/INFO_Abortion_WEB.pdf). 2. Creinin MD, Grossman DA. Medication abortion up to 70 days of gestation: ACOG practice bulletin, number 225. Obstet Gynecol 2020;136(4):e31-e47.

3. Kulier R, Kapp N, Gülmezoglu AM, Hofmeyr GJ, Cheng L, Campana A. Medical methods for first trimester abortion. Cochrane Database Syst Rev 2011;11: CD002855.

4. Costescu D, Guilbert E, Bernardin J, et al. Medical abortion. J Obstet Gynaecol Can 2016;38:366-89.

5. Mifeprex REMS Study Group. Sixteen years of overregulation: time to unburden mifeprex. N Engl J Med 2017;376:790-4.

6. Gissler M, Fronteira I, Jahn A, et al. Terminations of pregnancy in the European Union. BJOG 2012;119:324-32.

7. Baird B. Medical abortion in Australia: a short history. Reprod Health Matters 2015;23:169-76.

8. American College of Obstetricians and Gynecologists. Improving access to mifepristone for reproductive health indi- cations. June 2018 (https://www.acog.org/ clinical-information/policy-and-position -statements/position-statements/2018/ improving-access-to-mifepristone-for -reproductive-health-indications).

9. Gambir K, Garnsey C, Necastro KA, Ngo TD. Effectiveness, safety and acceptability of medical abortion at home versus in the clinic: a systematic review and meta-analysis in response to COVID-19. BMJ Glob Health 2020;5(12):e003934.

10. Grant K. Long-awaited abortion pill Mifegymiso makes Canadian debut. Globe and Mail. January 20, 2017 (https:// beta.theglobeandmail.com/news/ national/long-awaited-abortion-pill -mifegymiso-rolls-out-in-canada/ article33695167/?ref=http://www .theglobeandmail.com\&).

11. Gynuity Health Projects. Map of mifepristone approvals. June 2017 (http:// gynuity.org/resources/info/map-of -mifepristone-approvals/).

12. Health Canada. Regulatory decision summary: Mifegymiso. 2015 (https://cart -grac.ubc.ca/np-mifepristone-study/ regulatory-decision-summary-sbd _-mifegymiso-2015-health-canada/?login). 13. Munro S, Guilbert E, Wagner M-S, et al. Perspectives among Canadian physi- cians on factors influencing implementation of mifepristone medical abortion: a national qualitative study. Ann Fam Med 2020;18:413-21.

14. Health Canada. Mifegymiso: Health Canada updates prescribing and dispensing information for Mifegymiso. November 7, 2017 (https://hpr-rps.hres.ca/reg -content/regulatory-decision-summary -detail.php?lang=en\&linkID=RDS00294). 15. Guilbert ER, Hayden AS, Jones HE, et al. First-trimester medical abortion practices in Canada: National survey. Can Fam Physician 2016;62(4):e201-e208.

16. ICES. Mission, vision \& values (https:// www.ices.on.ca/About-ICES/Mission -vision-and-values).

17. Samiedaluie S, Peterson S, Brant R, Kaczorowski J, Norman WV. Validating abortion procedure coding in Canadian administrative databases. BMC Health Serv Res 2016;16:255.

18. World Health Organization. Women of reproductive age (15-49) population (thousands). April 12, 2021 (https://www .who.int/data/maternal-newborn-child -adolescent-ageing/indicator-explorer -new/mca/women-of-reproductive-age -(15-49-years)-population-(thousands)). 19. Saeed S, Moodie EEM, Strumpf EC, 
Klein MB. Segmented generalized mixed effect models to evaluate health outcomes. Int J Public Health 2018;63:547-51.

20. Bernal JL, Cummins S, Gasparrini A. Interrupted time series regression for the evaluation of public health interventions: a tutorial. Int J Epidemiol 2017;46:348-55. 21. Turner SL, Karahalios A, Forbes AB, et al. Creating effective interrupted time series graphs: review and recommendations. Res Synth Methods 2021;12:106-17. 22. Haukoos JSLR, Lewis RJ. Advanced statistics: bootstrapping confidence intervals for statistics with "difficult" distributions. Acad Emerg Med 2005;12:360-5. 23. Hategeka C, Ruton $\mathrm{H}$, Karamouzian M, Lynd LD, Law MR. Use of interrupted time series methods in the evaluation of health system quality improvement interventions: a methodological systematic review. BMJ Glob Health 2020;5(10):e003567. 24. Nelson BK. Statistical methodology. $\mathrm{V}$. Time series analysis using autoregressive integrated moving average (ARIMA) models. Acad Emerg Med 1998;5:739-44. 25. Wagner AK, Soumerai SB, Zhang F, Ross-Degnan D. Segmented regression analysis of interrupted time series studies in medication use research. J Clin Pharm Ther 2002;27:299-309.

26. Matheson FI, van Ingen T. 2011 Ontario marginalization index: technica document. Toronto: St. Michael's Hospi- tal, November 2017 (https://www .publichealthontario.ca/-/media/ documents/on-marg-technical.pdf?la=en), 27. Ammon Avalos L, Galindo C, Li D-K. A systematic review to calculate background miscarriage rates using life table analysis. Birth Defects Res A Clin Mol Teratol 2012;94:417-23.

28. Brown BP, Hebert LE, Gilliam M, Kaestner R. Association of highly restrictive state abortion policies with abortion rates, 2000-2014. JAMA Netw Open 2020; 3(11):e2024610

29. Ferris LE, McMain-Klein M. Smallarea variations in utilization of abortion services in Ontario from 1985 to 1992. CMAJ 1995;152:1801-7.

30. Berard V, Fiala C, Cameron S, Bombas T, Parachini M, Gemzell-Danielsson K. Instability of misoprostol tablets stored outside the blister: a potential serious concern for clinical outcome in medical abortion. PLoS One 2014;9(12):e112401.

31. Cleland K, Creinin MD, Nucatola D, Nshom M, Trussell J. Significant adverse events and outcomes after medical abortion. Obstet Gynecol 2013;121:166-71.

32. Chong $\mathrm{E}$, Shochet $\mathrm{T}$, Raymond $\mathrm{E}$, et al. Expansion of a direct-to-patient telemedicine abortion service in the United States and experience during the COVID-19 pandemic. Contraception 2021;104:43-8.

33. Aiken A, Lohr PA, Lord J, Ghosh N,
Starling J. Effectiveness, safety and acceptability of no-test medical abortion (termination of pregnancy) provided via telemedicine: a national cohort study. BJOG 2021;128:1464-74.

34. Jones HE, O'Connell White K, Norman WV, Guilbert E, Lichtenberg ES, Paul M. First trimester medication abortion practice in the United States and Canada. PLoS One 2017;12(10):e0186487.

35. Kulier R, Kapp N. Comprehensive analysis of the use of pre-procedure ultrasound for first- and second-trimester abortion. Contraception 2011;83:30-3.

36. Grimes DA. Estimation of pregnancyrelated mortality risk by pregnancy outcome, United States, 1991 to 1999. Am J Obstet Gynecol 2006;194:92-4.

37. Costescu D, Guilbert É. No. 360 - induced abortion: surgical abortion and second trimester medical methods. J Obstet Gynaecol Can 2018;40:750-83.

38. Devane C, Renner RM, Munro S, et al. Implementation of mifepristone medical abortion in Canada: pilot and feasibility testing of a survey to assess facilitators and barriers. Pilot Feasibility Stud 2019;5: 126.

39. Kortsmit K, Jatlaoui TC, Mandel MG et al. Abortion surveillance - United States, 2018. MMWR Surveill Summ 2020; 69(7):1-29.

Copyright $(2021$ Massachusetts Medical Society. 